\title{
1 Spatial metabolomics of in situ, host-microbe interactions
}

2

3 Benedikt Geier ${ }^{1}$; E. Maggie Sogin ${ }^{1}$; Dolma Michellod ${ }^{1}$; Moritz Janda ${ }^{1}$; Mario Kompauer ${ }^{3}$; Bernhard

$4 \quad$ Spengler $^{3} ;$ Nicole Dubilier ${ }^{1,2} ;$ Manuel Liebeke ${ }^{1 *}$

5

6

$7 \quad{ }^{1}$ Max Planck Institute for Marine Microbiology, Bremen, Germany

$8 \quad{ }^{2}$ MARUM, University of Bremen, Bremen, Germany

$9{ }^{3}$ Institute of Inorganic and Analytical Chemistry, Justus Liebig University Giessen, Giessen, Germany

10 *Corresponding author, email: mliebeke@ mpi-bremen.de, tel: +49 421 2028-822 


\section{Abstract}

Spatial metabolomics describes the location and chemistry of small molecules involved in metabolic phenotypes, defense molecules and chemical interactions in natural communities. Most current techniques are unable to spatially link the genotype and metabolic phenotype of microorganisms in situ at a scale relevant to microbial interactions. Here, we present a spatial metabolomics pipeline (metaFISH) that combines fluorescence in situ hybridization (FISH) microscopy and high-resolution atmospheric pressure mass spectrometry imaging (AP-MALDI-MSI) to image host-microbe symbioses and their metabolic interactions. metaFISH aligns and integrates metabolite and fluorescent images at the micrometer-scale for a spatial assignment of host and symbiont metabolites on the same tissue section. To illustrate the advantages of metaFISH, we mapped the spatial metabolome of a deepsea mussel and its intracellular symbiotic bacteria at the scale of individual epithelial host cells. Our analytical pipeline revealed metabolic adaptations of the epithelial cells to the intracellular symbionts, a variation in metabolic phenotypes in one symbiont type, and novel symbiosis metabolites. metaFISH provides a culture-independent approach to link metabolic phenotypes to community members in situ - a powerful tool for microbiologists across fields. 


\section{Introduction}

Across host-microbe interactions, metabolites are the immediate effectors underlying the basic principles of recognition, communication and manipulation between the symbiotic partners ${ }^{1-4}$. The exchange of metabolites is governed by the cellular organization of the symbiosis, such as the extra- or intracellular location of the microorganisms ${ }^{5}$. To maintain this spatial organization, hosts and microbes have developed unique metabolites, such as membrane constituents ${ }^{6}$, antimicrobials ${ }^{7,8}$ or recognition molecules $^{9,10}$, that are located at specific sites. The spatial metabolome describes this site-specific chemistry of the fine scale distribution of metabolites, including metabolic phenotypes of associated partners, subpopulations and chemical microenvironments ${ }^{11-13}$. Therefore, the visualization of the distribution of metabolites is central to understanding the interactions of microorganisms with animal and plant tissues, and within microbial consortia ${ }^{14,15}$.

Metabolomics allows the detection of thousands of metabolites from a homogenate of a complex community in a single mass spectrometry (MS) measurement ${ }^{16}$. However, the homogenization destroys the spatial organization of host-microbe associations, cells, and metabolites. Linking the spatial metabolome to the individual partners of a community is a major challenge for the study of host-microbe and microbe-microbe interactions ${ }^{17-19}$.

Mass spectrometry imaging (MSI) is a technique that allows the visualization of the spatial metabolome of close-to natural-state samples in situ ${ }^{14,15,20}$. However, MSI alone cannot reveal the taxonomic identity of community members. A well-established method for identifying individual members of microbial communities is fluorescence in situ hybridization (FISH), in which fluorescent probes hybridize to the $16 \mathrm{~S}$ rRNA gene of the targeted microorganisms ${ }^{19,21,22}$. To date, the only wellestablished MSI approach combined with FISH is nanoscale secondary ion mass spectrometry (nanoSIMS), which has become a powerful tool for linking isotope-based estimates of microbial activity to the identity of community members ${ }^{23,24}$. NanoSIMS does not allow the spatial imaging of metabolites. 
To image metabolites in the lower micrometer range, recent technical advances ${ }^{25-28}$ in matrix-assisted laser desorption ionization MSI (MALDI-MSI) $)^{29-31}$ provide an excellent tradeoff between breadth of detectable metabolites, spatial resolution and decreased destructivity. These advances could bridge the gap in resolution to FISH microscopy, making structures of metabolite images and single host cells or small microbial communities comparable. Consequently, combining high spatial resolution MALDIMSI with FISH would provide a spatial link between metabolic phenotypes and the taxonomic identity of microorganisms ${ }^{17,18}$. However, previous studies that combined MALDI-MSI with FISH, struggled with the destructivity of the MALDI laser when applying FISH after MALDI-MSI. They either applied the two methods to consecutive tissue sections of $12 \mu \mathrm{m}$ thickness ${ }^{5}$, or were limited to spatial resolutions above $50 \mu \mathrm{m}$ when applying both techniques to the same tissue surface ${ }^{17}$. Given these limitations, the combined approaches lack the resolution to sufficiently resolve the spatial metabolome of bacteria and most eukaryotic cells in three dimensions. We developed a MALDI-MSI and FISH pipeline capable of resolving the spatial metabolome of individual eukaryotic cells and bacterial microcolonies.

Most hosts and microbes are not yet culturable in their natural symbiotic association and visualizing how bacteria influence the host spatial metabolism is nearly impossible ${ }^{32}$. Therefore, we use Bathymodiolus puteoserpensis as an example for a non-culturable host-microbe symbiosis to demonstrate that our spatial metabolomics pipeline can be applied in situ. B. puteoserpentis is a deepsea mussel found at hydrothermal vents on the Mid-Atlantic Ridge. It lives in symbiosis with two types of intracellular gammaproteobacterial symbionts that provide it with nutrition ${ }^{33}$. One is a sulfur oxidizer (SOX) that gains energy through the oxidation of reduced sulfur compounds and hydrogen, and fixes $\mathrm{CO}_{2}$ into organic compounds. The other symbiont is a methane oxidizer (MOX) that uses methane as both an energy and a carbon source. The mussel hosts both mutualistic symbionts in specialized epithelial cells of the gill called bacteriocytes, reaching tremendous abundances of $10^{12}$ bacterial cells in a few centimeters of gill tissue ${ }^{34}$. The gill is structured into distinct regions of colonized and bacteria-free tissue $\mathrm{e}^{33,35}$ where the spatial metabolism and metabolic interactions are unknown. 
Here, we present our correlative imaging workflow for FISH microscopy after high resolution AP-

MALDI-MSI on the same tissue section. We were able to find genome-predicted phenotypes and link the spatial metabolome to the taxonomic identity of the associated partners of the host-microbe and microbe-microbe communities.

\section{Results and Discussion}

Our application of AP-MALDI-MSI and FISH to the same tissue section at a $3 \mu \mathrm{m}$ MSI-pixel size presents a significant advance to spatial metabolomics in microbiology. It enables the co-identification of metabolites and bacterial phylotypes at a scale relevant to microbial interactions (Fig 1a). We use the term 'phylotype' here as a proxy for bacterial species ${ }^{36}$.

To analyze the correlative fluorescence and metabolite images, we developed a FISH-based spatial metabolome binning approach after which we termed our imaging and analysis pipeline metaFISH. Our metaFISH pipeline consists of three modules: (I) Sample preparation and correlative imaging of AP-MALDI-MSI before FISH (Fig. 1a); (II) Processing of the correlative imaging data, including a precise image alignment (Fig. 1b) and (III) A statistical analysis using our FISH-based spatial metabolome binning to spatially assign metabolite groups to fluorescence signals of host and symbionts (Figs 1c, d and 2c). Our pipeline provides correlation values indicating which metabolites correlate to host- or symbiotic tissues.

We applied metaFISH to investigate the symbiosis between the deep-sea mussel, B. puteoserpentis, and its intracellular microbial community (Fig. 2a). Using this unculturable model, we linked spatial metabolomes in the gill tissue to both the host and its bacteria. Our spatial metabolome data showed the mussel's intracellular bacteria alter the lipid composition in the symbiotic organ. Furthermore, we applied our approach to visualize metabolic heterogeneity within one single $16 \mathrm{~S}$ rRNA phylotype ${ }^{33}$. Finally, using our pipeline in a discovery driven approach, we identified a novel group of metabolites 


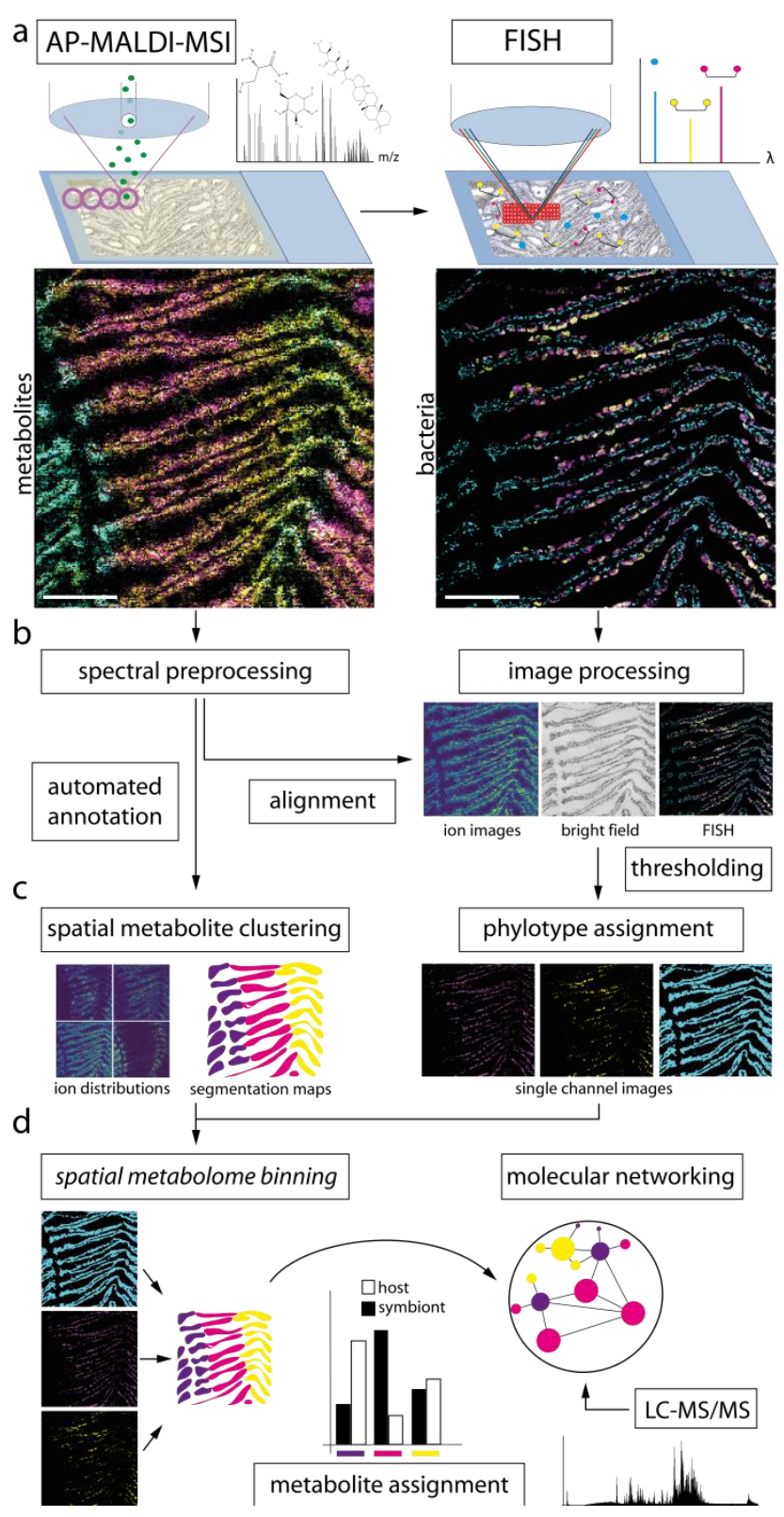
pipeline (metaFISH). a, Metabolite heat-maps from high-resolution AP-MALDI-MSI (overlay of three ions in cyan, yellow and magenta at a pixel size of $3 \mu \mathrm{m}$ ) acquired before fluorescent in situ hybridizations (FISH) of the same tissue section (example image showing DAPI-stained host nuclei in cyan and two types of FISHed bacterial endosymbionts in magenta and yellow). Scale bars: $150 \mu \mathrm{m}$. b, Spectral pre-processing, annotation, image alignment of AP-MALDI-MSI and FISH images and background subtraction from microscopy data. c, Spatial clustering of AP-MALDI-MSI data and image processing of FISH signals. d, Metabolite assignment to host and symbionts. FISH-based spatial metabolome binning results (MF-ratio) can then be used to visualize submetabolomes host and bacteria in molecular networks and to guide LC-MS/MS metabolite identification. 


\section{Metabolite assignment to bacteria-rich and bacteria-free tissue through spatial cross}

\section{correlations}

Spatial clustering of the AP-MALDI-MSI data revealed spatial partitioning of the gill metabolome.

Using metaFISH, we could categorize spatial metabolite clusters into bacteria-colonized and bacteriafree submetabolomes. After preprocessing of the raw AP-MALDI-MSI spectra, our MS data included 2506 metabolite images across 54,289 pixels. Using a spatially aware clustering approach ${ }^{37}$, we grouped all molecule distributions across the gill into seven clusters with distinct biochemistry (Fig. 2c, d, Supplementary Fig. 6). The spatial clustering also separated chemical background signals (cluster 1 and 3) from the tissue-associated metabolome (cluster 2, 4, 5, 6)(7; Supplementary Fig. 7), as confirmed by ion map distributions and compound annotations. This chemical noise is common in AP-MALDI-MSI as it originates from the matrix, a crystalline layer applied to the sample surface to assist ionization. Therefore, we could exclude all ions from the background clusters 1 and 3 from further analysis of tissue metabolites.

With metaFISH, we calculated a ratio (MF-ratio) from the relative overlap between metabolite cluster and fluorescent signals, which we used as an indicator, if a cluster was linked to the mussel host or its symbiotic bacteria (Fig. 2c) (Supplementary text 4 and Supplementary Table 1). Our metaFISH analysis found two host- (MF-ratio > 1) and three symbiont-associated (MF-ratio < 1) submetabolomes (Fig. 2c, d).

The precise FISH-image correlations allowed us to classify clusters as bacterial sub-metabolomes (e.g. cluster 6 and 5) n(Supplementary Fig. 7 and 9) even if they contained few pixels that were scattered throughout the tissue. Such visually random metabolite- and cluster distributions would have been considered unstructured noise ${ }^{38}$ by stand-alone AP-MALDI-MSI analyses or visual inspection. Supporting the identification of heterogeneous, low-signal metabolite patterns through label-specific correlative techniques will be crucial to differentiate noise from meaningful biological signals when moving towards single cell metabolomes of eukaryotes and bacteria. 
a

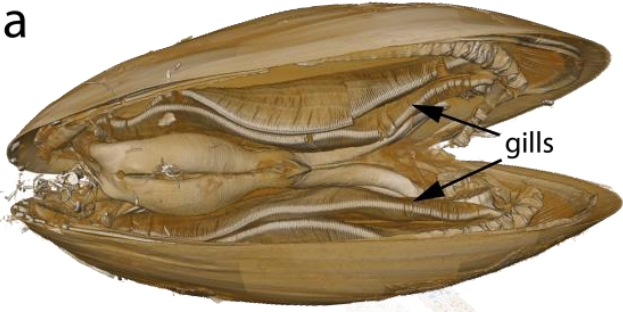

SOX
- MOX
host nucleus

- intercalary cell

bacteriocyte

1. ciliated edge cell

C

MSI Cluster \% of all symbiont or host tissue FISH signals Group

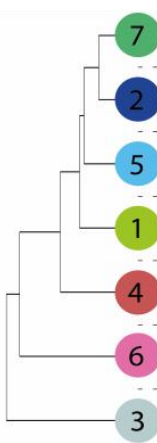

158

159

160

161

162

163

164

165

166

167

168

169

170

171

172 associated with each cluster

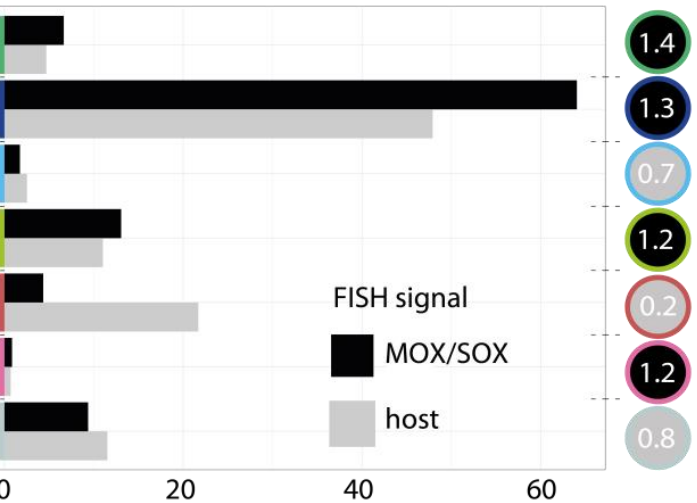

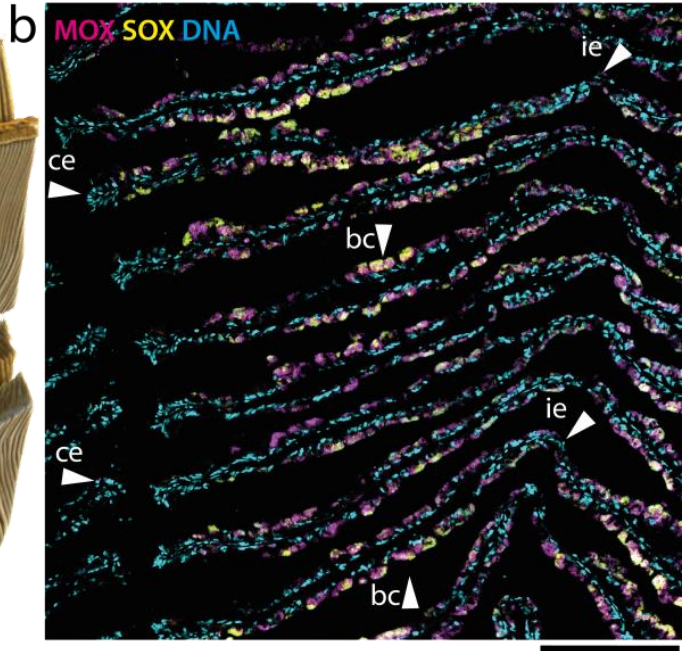

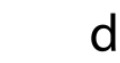

d
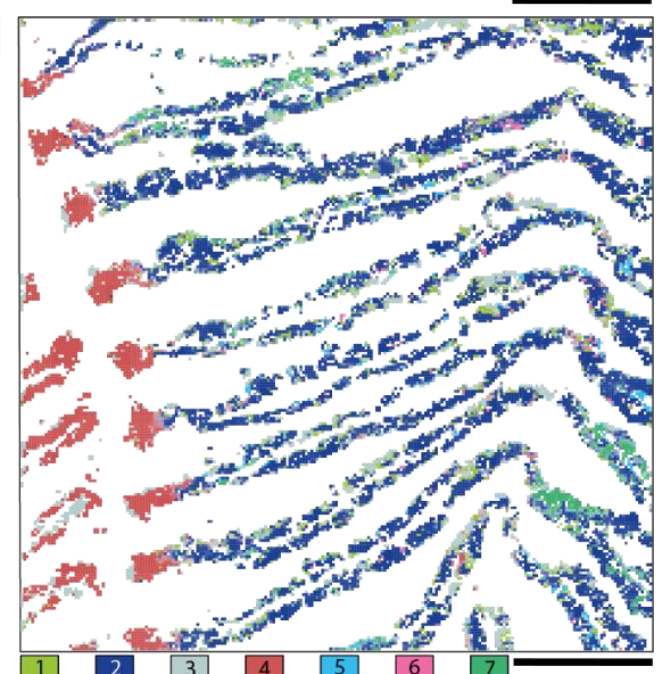

Fig. 2 | Spatial metabolome binning based on FISH signals in the symbiotic organ of B. puteoserpentis.

a, Micro-computed tomography 3D model of a Bathymodiolus deep-sea mussel (Supplementary text 3), virtual dissection of the gill and horizontal sectioning plane through gill filaments showing the symbiont-containing tissue. Schematic of the main cell types in the gills: symbiont-free ciliated edge cells, bacteriocytes with methane-oxidizing symbionts (MOX, magenta) and sulfur-oxidizing symbionts (SOX, green), intercalary cells. b, Fluorescence in situ hybridization (FISH): CMY overlay shows magenta channel, MOX; yellow channel, SOX; cyan channel, host DNA stain (ce, ciliated edge; ie, forming inner edge; bc, bacteriocyte). c, Hierarchical k-means clustering of the AP-MALDI-MSI data and FISH-based spatial metabolome binning represented as MF-ratios assigning cluster groups 1-7 to the host or symbionts (Supplementary Table 1). d, Segmentation maps of spatial clusters with their colors corresponding to c. Scale bars in b and d: $150 \mu \mathrm{m}$. 


\section{Visualization of distinct metabolic phenotypes of the endosymbiotic MOX}

The imaging of metabolite production can reveal phenotypic heterogeneity, for example within clonal communities that co-occur in the same microenvironment. ${ }^{11}$. Visualization of the micro-scale metabolome and community structure allowed us to study the in situ phenotypes of the endosymbiotic MOX. Our metaFISH pipeline revealed variations in the spatial metabolome of the MOX phylotype. We identified hopanoids as a distinct group of MOX-specific metabolites in the metaFISH-classified bacterial clusters 6 and 7 (Supplementary Fig. 11 and Supplementary table 8). Hopanoids are lipids commonly found in membranes of methane-oxidizing bacteria. In bulk-measurements, they are often used as biomarkers ${ }^{6}$ and we therefore used hopanoids as a proxy to locate the MOX symbionts in B. puteoserpentis. Unexpectedly, our micrometer-scale hopanoid maps showed a patchy distribution of single hopanoids compare to the 16S rRNA FISH signals of the MOX symbionts, and revealed clear differences in hopanoids among the MOX symbionts of a single host individual (Fig. 3, Supplementary Fig. 11). Our spatial clustering analyses supported our imaging analyses, and showed significant differences in the distribution aminobacteriohopane-triol in cluster 6 and bacteriohopanetetrol in cluster 7 (Fig. 3d).

To find more hopanoids that were not clustered we visualized the AP-MALDI-MSI data with a chemical networking approach. During MSI data acquisition, unfragmented metabolites are detected, which allows us to translate exact mass differences $(<5 \mathrm{ppm})$ between two compounds into defined chemical modifications (e.g. hydroxylation, alkylation; see full list in Supplementary Table 2) ${ }^{39}$. These modifications can be visualized as edges, connecting individual metabolites represented as nodes in a network $^{39}$. We highlighted spatial clusters and compound annotations in the AP-MALDI-MSI network, which showed two additional hopanoids, aminobacteriohopane-tetrol and anhydrobacteriohopane-tetrol directly connected to the two clustered hopanoids (Fig. 3d, Supplementary text 3, Supplementary Fig. 11).

The molecular network in fig. $3 \mathrm{~d}$ showed that a single hydroxyl group that differed between aminobacteriohopane-triol and aminobacteriohopane-tetrol explained the different distributions between these two metabolites (Fig. 3b, c). Both hopanoids were patchily distributed, with 
200 aminobacteriohopane-tetrol located towards the outer edge of the gills, and aminobacteriohopane-triol,

201 concentrated in individual bacteriocytes, in the center of the gills (Figs 3a, b, and c). With our

202 correlative imaging pipeline we could show that different hopanoid phenotypes are expressed by a

203 single symbiont phylotype and differ across single epithelial gill cells. Therefore, metaFISH could be

204 used to test the phenotypic heterogeneity in the distribution of biomarker molecules. Microbial

205 communities are known to change their hopanoid composition across meter long oxygen gradients ${ }^{40}$.

206 The gradual change of the two hopanoids (Fig. 3c) from the edge to the center of the gills could reflect

207 a phenotypic adaptation of the intracellular MOX symbionts to such micro-scale gradients. 
bioRxiv preprint doi: https://doi.org/10.1101/555045; this version posted February 19, 2019. The copyright holder for this preprint (which was not certified by peer review) is the author/funder, who has granted bioRxiv a license to display the preprint in perpetuity. It is made available under aCC-BY-NC-ND 4.0 International license.

\section{a}

35-aminobacteriohopane-

32,33,34-triol

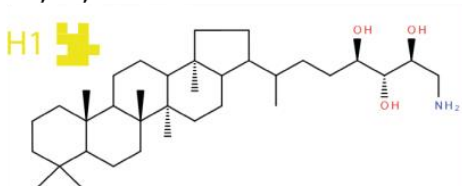

35-aminobacteriohopane-

31,32,33,34-tetrol

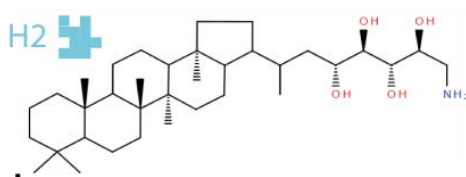

b

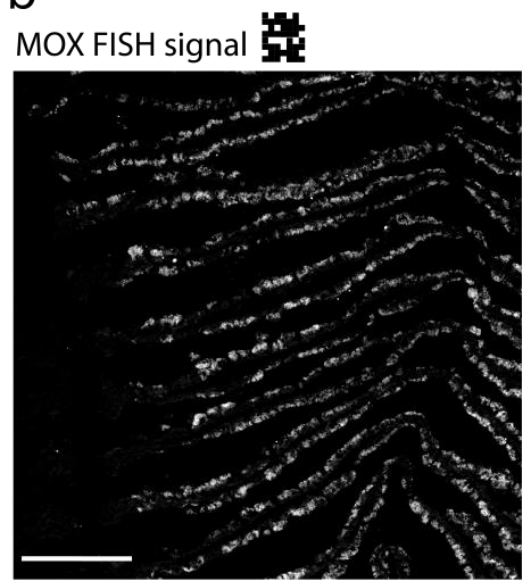

C
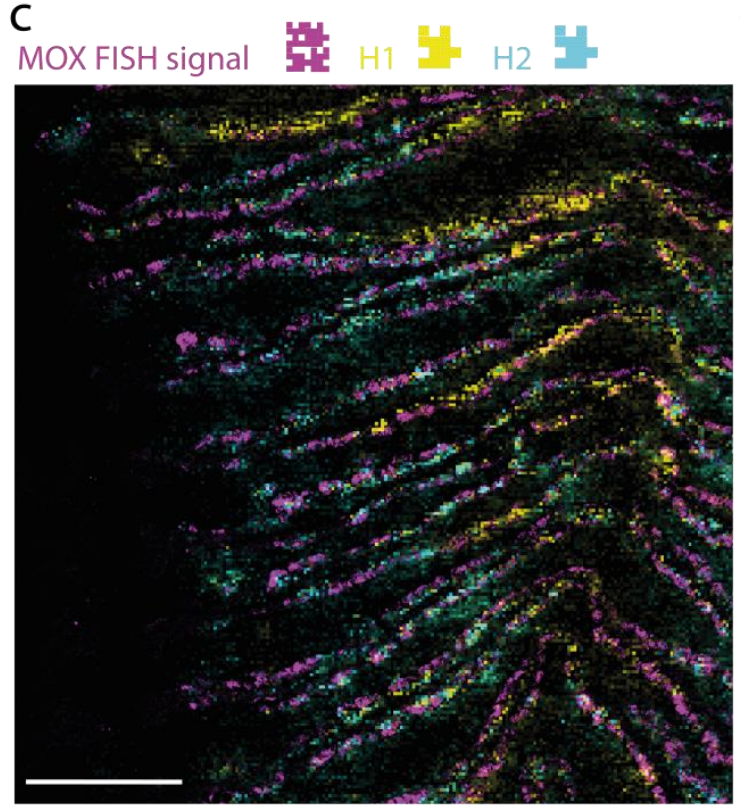
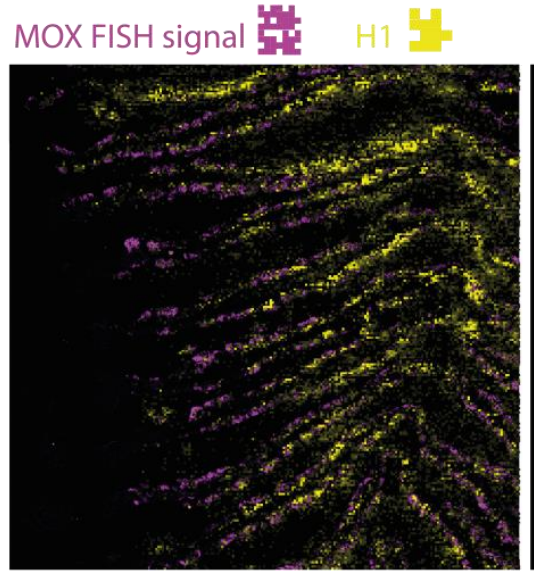

d

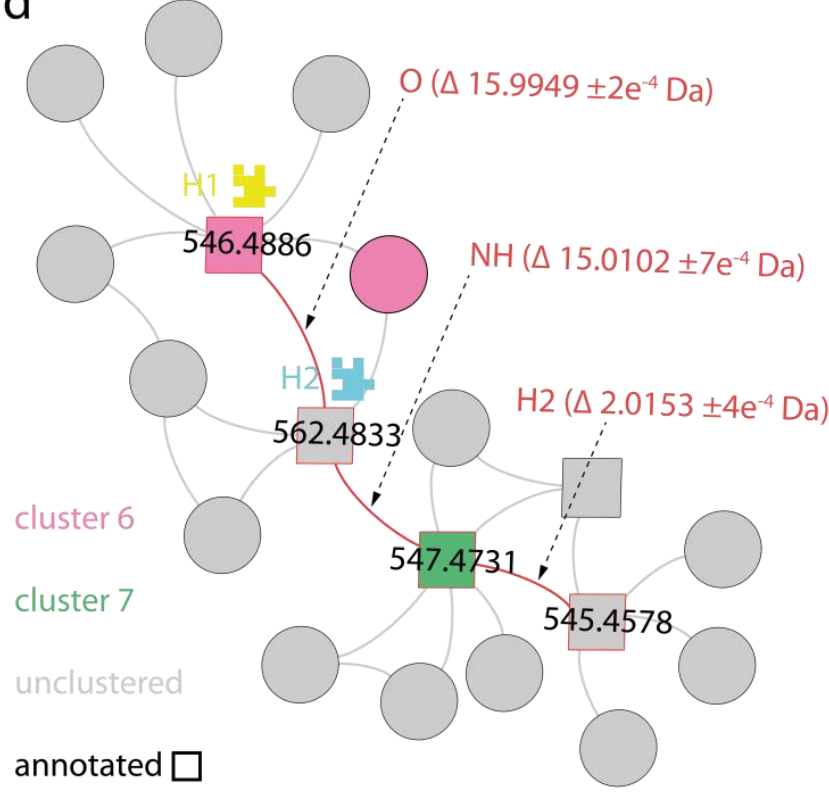

Fig. 3 | Micrometer-scale metabolic heterogeneity of MOX symbionts in the gill. a, Chemical structures and ion maps of the two most prominent hopanoids (H1, yellow and H2, cyan). b, FISH signal of the hopanoid-

211 producing, methane-oxidizing symbiont (grey scale) and the ion maps of each hopanoid, H1 (yellow) and H2

212 (cyan) overlaid onto the FISH signal of the methane-oxidizing symbiont (magenta). c, Minor overlap (white pixels) between the two abundant hopanoids: 35-aminobacteriohopane-32,33,34-triol (yellow) and 35- 
aminobacteriohopane-31,32,33,34-tetrol (cyan) on top of the MOX signal (magenta). Both are produced by an identical 16S rRNA phylotype of the methane-oxidizing symbiont (magenta) and show strong spatial metabolic heterogeneity. d, Integration of spatial metabolome binning data and annotation of metabolites in a molecular network shows that the major spatial changes (colored nodes, spatial cluster 6 red, cluster 7 green) are associated with minor chemical side-chain modifications (red edges: gain/loss of $\mathrm{O}, \mathrm{H}_{2}$ and an amine group) between the annotated hopanoids (square nodes, red edges). Identification of bacteriohopane-32,33,34,35-tetrol ( $\mathrm{m} / \mathrm{z}$ 547.4731) and 31-hydroxy-32,35-anhydro-bacteriohopane-tetrol (m/z 545.4578). Scale bars: $150 \mu \mathrm{m}$.

\section{Metabolic landscape of the symbiotic organ}

In addition to functioning as a respiratory organ, the gill of $B$. puteoserpentis has acquired functions of a bioreactor, where the tremendous symbiont biomass ${ }^{34}$ can grow under regulated conditions. Despite the well-known host-symbiont community structure of colonized and bacteria-free regions ${ }^{33,35}$, the spatial metabolome underlying the symbiotic organ is unknown. Many metabolites are related to each other chemically and spatially because they are involved in similar metabolic pathways and share biochemical reactions. We explored these chemical relations in our AP-MALDI-MSI networking approach where we integrated all 2506 detected molecules and highlighted the host- and symbiontassociated clusters (Fig. 4a).

The overall chemical space is divided into two main sub-networks separating host- and symbiontassociated metabolites (clusters 2, 4, 5, 6, and 7) from background matrix signals (clusters 1 and 3) (Fig. 4a, Supplementary Figs 7 and 10). Using the MSI metabolite annotation platform Metaspace ${ }^{41}$ annotations show that the tissue-associated metabolites were annotated as phospholipids, such as phosphatidylcholines (PC), which are the major membrane components in eukaryotic cells ${ }^{42}$ (clusters 2 and 4; Fig. 4b and Fig.4c). The PC(36:2), $\mathrm{C}_{44} \mathrm{H}_{84} \mathrm{NO}_{8} \mathrm{P}+\mathrm{H}^{+}, m / z 786.6012$ and the $\mathrm{PC}(32: 1)$,

$\mathrm{C}_{40} \mathrm{H}_{78} \mathrm{NO}_{8} \mathrm{P}+\mathrm{H}^{+}, m / z, 732.5543$, (Fig. 4c, Supplementary Figs 14, 16 and 17 and Supplementary table 8) were homogeneously distributed along each filament. This distribution was shared by over $60 \%$ of the ions in cluster 2, which could represent a baseline metabolomic signature of the gill tissue.

Most metabolites in our dataset from the largest bacteria-specific submetabolome (cluster 7) were annotated as triglycerides (TG) and were distributed similar to the TG(48:3), $\mathrm{C}_{51} \mathrm{H}_{92} \mathrm{O}_{6}+\mathrm{Na}^{+}, m / z$ 823.6794, and TG(52:3), $\mathrm{C}_{55} \mathrm{H}_{100} \mathrm{O}_{6}+\mathrm{K}^{+}, m / z 895.7142$ (Fig. 4d). These triglycerides formed a distinct 
chemical sub-network, separated from the main phospholipid sub-network containing ions of cluster 2 and 4 (Fig. 4a and Supplementary Fig. 10). The separation of phospholipids and triglycerides in the chemical networks reflects functional difference of these lipids in living cells. Phospholipids are membrane-bound whereas triglycerides are storage and transport lipids ${ }^{43}$ and synthesized from the turnover and degradation of phospholipid membranes ${ }^{44}$. The digestion of bacteria inside bacteriocytes requires the breakdown of the bacterial phospholipid membranes ${ }^{45}$. Consequently, our observed spatial submetabolome containing high triglyceride abundances in the bacteriocyte region could show metabolites originating from the digestion of the intracellular symbionts by the host.

In the bacteria-free ciliated edge tissue, we localized a phosphonate lipid, phosphonoethanolamine ceramide PnE-Cer(34:2), $\mathrm{C}_{36} \mathrm{H}_{72} \mathrm{~N}_{2} \mathrm{O}_{5} \mathrm{P}, m / z 643.5170$ (Fig. 4b and Supplementary Figs 12 and 13). Phosphonates are a under-studied class of metabolites that serve as a phosphorus and nitrogen source for some marine bacteria ${ }^{46,47}$. The PnE-Cer(34:2) is highly concentrated in the ciliated edge and in the center of the gills. We hypothesize that the accumulation of this lipid represents a potential niche for phosphonate degraders. In closely related Bathymodiolus species ${ }^{35}$, epibionts that colonize the ciliated edge possess the genes required for the degradation of phosphonates ${ }^{48}$.

We found host metabolites in cluster 4 that were highly abundant in the symbiont-free ciliated edge and decreased gradually towards the symbiont-colonized tissues including the center of the gills (Fig. 2b and Supplementary Figs 7 and 15). Within this group of spatially similar metabolites, we identified $\mathrm{PC}(34: 3), \mathrm{C}_{42} \mathrm{H}_{78} \mathrm{NO}_{8} \mathrm{P}+\mathrm{H}^{+}, m / z$ 756.5536(Fig. 4b and Supplementary Fig. 15). Unlike PnECer(34:2), $\mathrm{PC}(34: 3)$ is negatively correlated to the presence of bacteria. PCs can be synthesized by only $10 \%$ of bacteria, and some intracellular bacteria have been shown to scavenge PCs as a choline source $^{42}$. We hypothesize that distribution patterns like that of $\mathrm{PC}(34: 3)$ indicates either a reduction in metabolite synthesis or degradation by the intracellular symbionts in the bacteriocytes. 


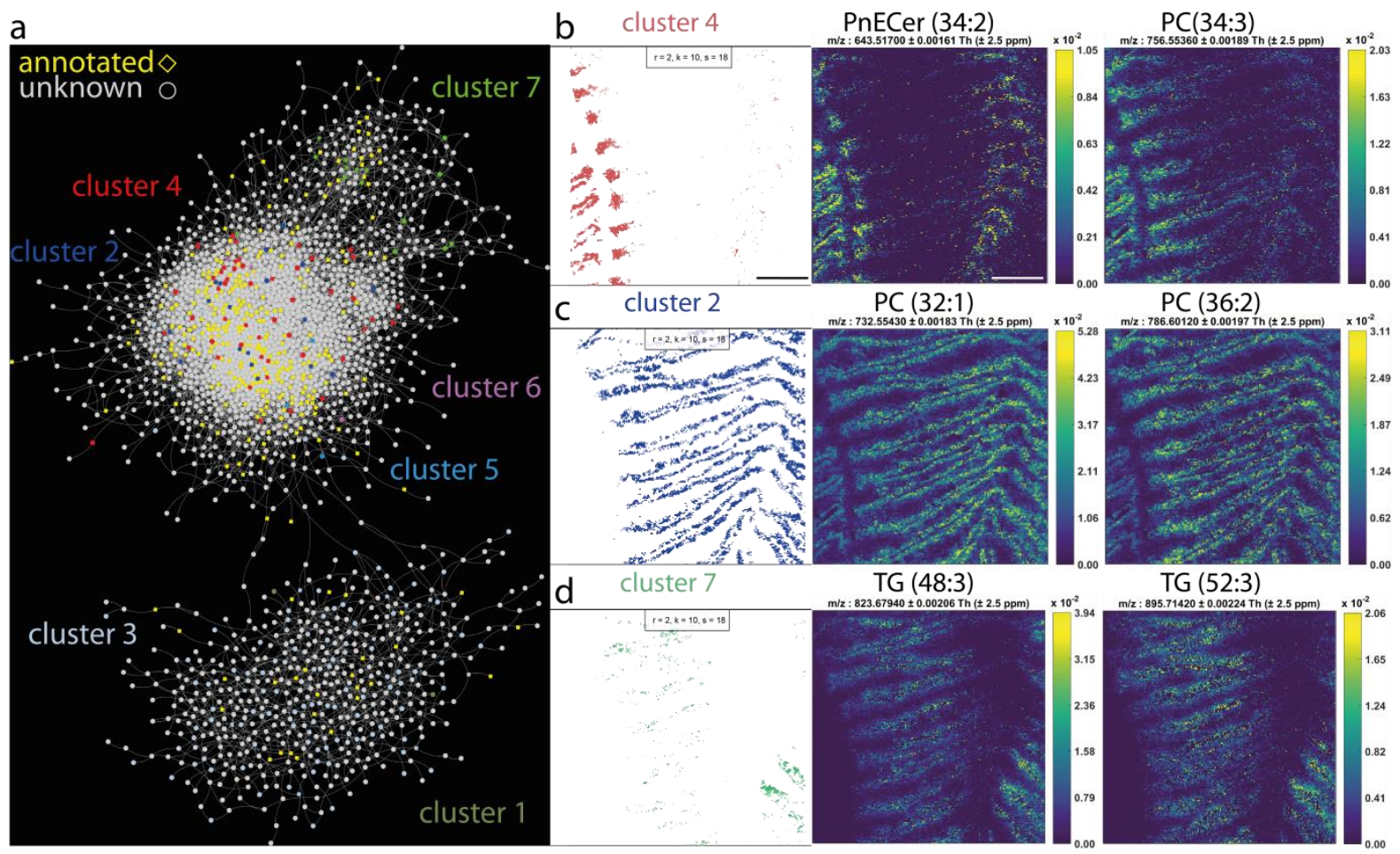

Fig. 4 | Metabolic landscape of the symbiotic organ. a, Molecular network visualizing all detected molecules, representing their spatial and potential chemical relationships. This MS1 based network facilitates data exploration by showing how similarly distributed metabolites (classified as clusters) and unclassified ions are related chemically. Nodes, $\mathrm{m} / \mathrm{z}$ values of unfragmented parental ions; edges, defined molecular transformations calculated from $\Delta \mathrm{m} / \mathrm{z}$; nodes colored with respect to cluster membership, yellow if not clustered; diamond shaped nodes match annotations $\pm 10 \mathrm{ppm}$; round grey nodes are unknown metabolites. b-d, Major spatial submetabolomes visualized as segmentation maps, and two representative metabolite distributions of annotated and identified metabolites. b, PnECer(34:2) is abundant in the ciliated and inner edges, whereas PC(34:3) shows a high abundance only in the ciliated edge and decreases towards the center of the gills. c, PC(32:1) and PC (36:2) are homogeneously distributed throughout the gill filaments. d, TG(48:3) and TG(52:3) show a high abundance only in the bacteria-rich regions and are absent in the bacteria-free ciliated edge and bacteria-poor inner edge. Scale bar $150 \mu \mathrm{m}$.

\section{Discovery of new metabolites specific to the mussel-MOX symbiosis}

Invertebrate host-microbe symbioses are rich in specialized bioactive compounds ${ }^{49}$. A key challenge in natural product discovery is determining which of the thousands of measured metabolites serve regulatory roles or are metabolically active in microbial interactions ${ }^{50}$. MetaFISH provides a powerful tool to screen for metabolites involved in these chemical interactions at the host-microbe interface. 
To demonstrate the potential of metaFISH for molecular prospecting, we identified the ion $m / z 869.5375$ as the metabolite with the highest co-localization with the FISH signal within bacterial submetabolome (cluster 2) after visual inspection (Fig. 5a and Supplementary Fig. 32). Using our APMALDI-MSI networking approach (Fig. 4a), we found four similarly distributed metabolites linked to the clustered ion $\mathrm{m} / \mathrm{z} 869.5375$ through alkane chain length transformations $(\mathrm{m} / \mathrm{z} 813.4736, \mathrm{~m} / \mathrm{z}$ 815.4902, $m / z 841.5062$ and $m / z$ 843.5215shown in Fig. 5d) (Supplementary Figs 18 and 19 and Supplementary Table 4). LC-MS/MS fragmentation spectra confirmed each metabolite as a homolog of $m / z$ 869.5375. For all five metabolites, the fragmentation pattern showed a terminal fatty acid with variable lengths (loss of $\mathrm{C}_{16}-\mathrm{C}_{20}$ shown in Fig. $5 \mathrm{~b}$ ) and a pentose moiety (loss of $\Delta m / z$ 132.04 Da shown inFig. 5e) (Supplementary Figs 20-30 and Supplementary table 8). The core of those metabolites (without pentose moiety and fatty acid) was identified by exact mass determination using magnetic resonance mass spectrometry (MRMS) as $\mathrm{C}_{21} \mathrm{H}_{25} \mathrm{~N}_{6} \mathrm{O}_{3}(\mathrm{~m} / z 409.198213 \pm 0.128 \mathrm{ppm}$, Supplementary table 9). The similarity of ion fragmentation was supported by LC-MS/MS based molecular networking with the Global Natural Products Social Molecular Networking platform (GNPS) (Supplementary Fig. 20 and Supplementary text 3$)^{51}$. We termed the metabolites $\mathrm{m} / \mathrm{z}$ 813.4736, $\mathrm{m} / \mathrm{z} 815.4902, \mathrm{~m} / \mathrm{z} 841.5062, \mathrm{~m} / \mathrm{z} 843.5215$ and $\mathrm{m} / \mathrm{z} 869.5375$ as the "800 group" based on their similar structures and spatial distributions (Supplementary Fig. 19 and Supplementary table 4). In our AP-MALDI-MSI network, we connected the metabolite $m / z$ 577.2604 (from now on termed 577) to all metabolites of the 800 group with edges matching mass differences of different fatty acids (Supplementary Fig. 18 and Supplementary table 8). We could verify that the metabolite 577 was a variant of the 800 group without the fatty acid moiety, based on their fragmentation patterns, UV absorbance (Figs 5d, 5e and Supplementary Figs 21, 22, 29, 30 and 31, Supplementary table 4) and their shared core sum formula $\mathrm{C}_{21} \mathrm{H}_{25} \mathrm{~N}_{6} \mathrm{O}_{3}$ and $\mathrm{C}_{21} \mathrm{H}_{29} \mathrm{~N}_{6} \mathrm{O}_{5}\left[\mathrm{M}+2 \mathrm{H}_{2} \mathrm{O}\right]^{+}$(445.219322 \pm 0.164 ppm; Supplementary table 9) as identified by MRMS. Unlike the 800 group, the metabolite 577 was abundant in gill tissue regions free of bacteria (cluster 5, metaFISH ratio $=0.7$, Fig. 5b). Interestingly, overlaying the distributions (Fig. 5c) revealed that 577 in the host tissue surrounded the bacteriocytes in which the homologs of the 800 group are abundant. We hypothesize that the metabolite 577 could 
312 be either a compound precursor, where long chain fatty acids are added through esterification resulting

313 in members of the 800 group, or 577 could be a hydrolysis product of the 800 group, where the fatty

314 acids are cleaved and 577 is accumulated in the host tissue. Our findings suggest novel chemical

315 structures involved in the Bathymodiolus symbiosis, for which we found no database annotations or

316 metabolites with similar fragmentation patterns across datasets in GNPS using its dereplication

317 pipeline (Supplementary text 3$)^{51}$.

318 To further investigate the role of this metabolite group, we used LC-MS/MS to screen 11 other

319 symbiotic, deep-sea mytilid species (seven Bathymodiolus, three Gigantidas and one Vulcanidas)

320 containing varying combinations of SOX and MOX symbionts from 9 different deep-sea vents

321 (Supplementary Fig. 33 and Supplementary Table 3) ${ }^{52}$. Our screening revealed that both the 800 and

322577 metabolites only occurred in Bathymodiolus and Gigantidas mussels when the MOX symbiont

323 was present. In contrast, we also screened a free-living MOX relative (Methyloprofundus sedimenti) ${ }^{53}$,

324 which did not contain either the 577 or the 800 group (Supplementary Table 3).

325 Although the detailed structure, function and symbiotic partners responsible for the synthesis are still

326 to be determined, these data indicate this metabolite group is involved in the symbiosis between deep-

327 sea mussels and MOX symbiont (Supplementary Fig. 33 and Supplementary Table 3). Our metaFISH

328 pipeline provides a valuable contribution to conventional secondary metabolite screening for

329 metabolites that spatially and chemically mediate within host-microbe interactions. 


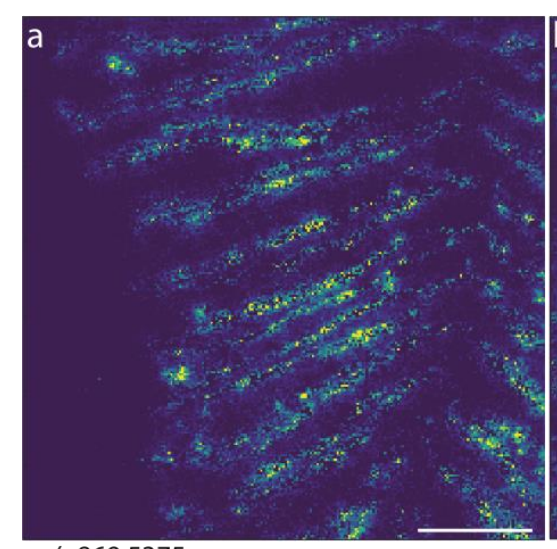

$$
m / z 869.5375
$$

$$
\text { d }
$$

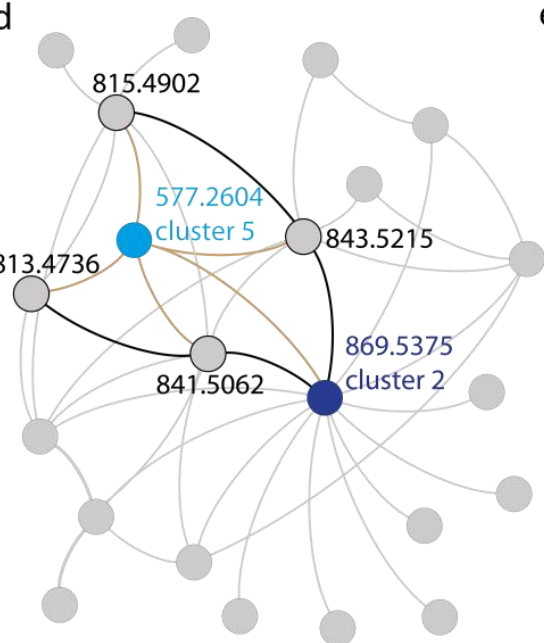

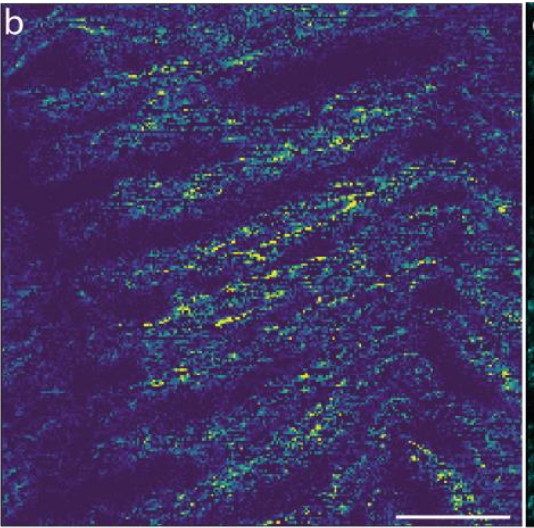

$m / z 577.2604$

e

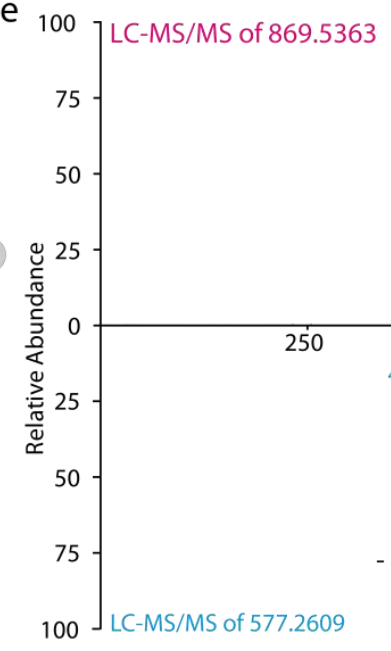

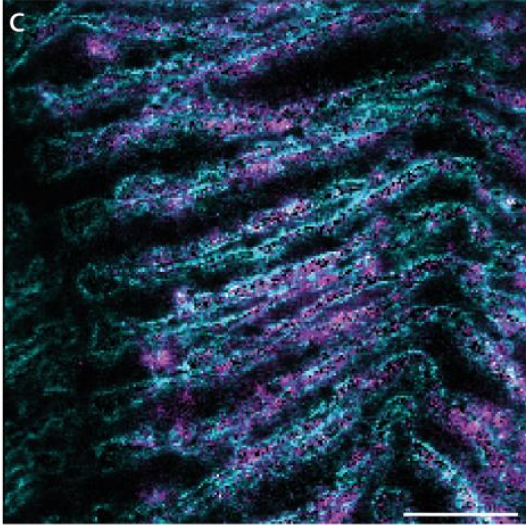

$\mathrm{m} / \mathrm{z} 869.5375 \mathrm{~m} / \mathrm{z} 577.2604$

869.5363

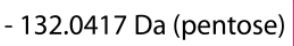

Fig. 5 | Discovery of new symbiosis metabolites specific to the mussel-MOX symbiont interaction.

a, Metabolite $m / z 869.5375$ is strongly correlated with the symbiont communities and $\mathbf{b}, m / z 577.2604$ with host tissue. c, MSI overlay of $m / z 869.5375$ (magenta) and $m / z 577.2604$ (cyan). Scale bars: $150 \mu$ m. d, MALDI-MSI sub-network showing "800 group” ions around $m / z, 577.2604$ (grey nodes: unclustered ions). Black edges linking bacteria-correlated metabolites of the "800 group" represent mass differences of alkyl chain lengths. Brown edges correspond to mass differences of fatty acids, present in the 800 group-metabolites and absent in the hostcorrelated metabolite (577.2604, cyan node) (Supplementary Fig. 16). e, Characteristic LC-MS/MS fragmentation pattern for $m / z 869.5363$ (magenta) and for $m / z 577.2609$ (cyan). $m / z 869.5363$ (upper spectrum, magneta) loses a pentose (black) and a fatty acid (brown). $\mathrm{m} / \mathrm{z} 577$ (lower spectrum, cyan) only loses a pentose (black). Fragment ion $m / z, 541.2402$ in the upper spectrum represents the host metabolite $m / z, 577.2609-2 \mathrm{H}_{2} \mathrm{O}(\Delta$ 36.0207 Da). 


\section{Conclusion}

347 Linking the spatial metabolome to individual partners of multimember communities, such as host-

348 microbe associations, provides insights into their metabolic interactions. Metabolite imaging coupled

349 with phylotype-specific labeling creates a direct link between the metabolism of a microorganism and

350 its identity. The potential for additional labeling methods, for example of individual genes ${ }^{54}$ or

351 transcripts ${ }^{55}$, would provide an even higher resolution of genotypic and phenotypic correlations.

352 With metaFISH, MALDI-MSI with FISH microscopy can link metabolomes to single eukaryotic host

353 cells or micro-scale communities, and given the fast pace of technical improvements in spatial

354 resolution, metabolite imaging will allow for discrimination at the scale of single bacteria. However,

355 independent of the spatial resolution, metabolite imaging will rely on correlative approaches to

356 identify the taxonomic identities underlying the vast phenotypic heterogeneity in eukaryotic and

357 prokaryotic cells.

358 Researchers are beginning to apply MALDI-MSI to samples from natural environments beyond

359 controlled laboratory conditions. Plant and animal tissues are commonly associated with microbes,

360 which can significantly impact the metabolome of the host. If these microbes are overlooked, their

361 contribution to the spatial metabolome will remain obscured as a host phenotype.

362 Applied to host-pathogen interactions, our metaFISH pipeline could be used to simultaneously

363 visualize phenotypes of microbial pathogens and the metabolic immune response of the host. For

364 instance, this could include stage specific phenotype changes during tubercle formation of

365 Mycobacterium tuberculosis ${ }^{56}$ or the transition from a recurrent to a chronic infection with

366 Pseudomonas aeruginosa ${ }^{57}$ during cystic fibrosis.

367 We envision that metaFISH will extend the capabilities of modern meta-omics for linking the identity

368 of microbial community members to their metabolism and enable us to decipher the chemical

369 language of microbes among each other and with their hosts. 


\section{Acknowledgements}

372

373

374

375

376

377

378

379

380

381

We would like to thank the crew and captains of the scientific vessels Meteor (M64 M114 M126),

Nautilus (Na 58), Sonne (SO253) and Atlantis (AT26-10, AT21-02) and their ROV pilots that helped us collect our extensive sample set. We thank Miguel Ángel González Porras for advice during FISH experiments, Merle Ücker for support in the lab, and Stephanie Markert for providing the Bathymodiolus thermophilus samples used for LC-MS/MS. We thank Matthias Witt from Bruker Daltonik GmbH for the exact mass measurements using scimaX ${ }^{\mathrm{TM}}$ MRMS. This work was funded by the Max Planck Society, the DFG Cluster of Excellence 'The Ocean in the Earth System' at MARUM (University of Bremen), a Gordon and Betty Moore Foundation Marine Microbiology Initiative Investigator Award through grant GBMF3811 to ND and a European Research Council Advanced Grant (BathyBiome, Grant 340535). For instrumental development, financial support by the Deutsche Forschungsgemeinschaft, DFG under grant Sp314/13-1 is gratefully acknowledged.

Conflict of interest. BS is a consultant and MK is an employee of TransMIT GmbH, Giessen, Germany. All other authors declare no conflict of interest.

Data availability. Download links for the raw files of fluorescence microscopy data, AP-MALDIMSI and on-tissue AP-MALDI-MS/MS data and LC-MS/MS data are provided in the Supplementary Text 3. Data, analyzed with the online annotation and networking platforms Metaspace and GNPS can also be accessed through the provided links in the Supplementary Text 3.

\section{References}

1 Cleary, J. L., Condren, A. R., Zink, K. E. \& Sanchez, L. M. Calling all hosts: Bacterial communication in situ. Chem 2, 334-358, doi:10.1016/j.chempr.2017.02.001 (2017).

2 Song, C. X. et al. Molecular and chemical dialogues in bacteria-protozoa interactions. Sci RepUk 5, doi:Artn 12837

10.1038/Srep12837 (2015).

3 Garg, N. et al. Spatial Molecular Architecture of the Microbial Community of a Peltigera Lichen. Msystems 1, doi:UNSP e00139 
400

401

402

403

404

405

406

407

408

409

410

411

412

413

414

415

416

417

418

419

420

421

422

423

424

425

426

427

428

429

430

431

432

433

434

435

436

437

438

439

440

441

442

443

444

445

446

447

448

10.1128/mSystems.00139-16 (2016).

4 Chagas, F. O., Pessotti, R. D., Caraballo-Rodriguez, A. M. \& Pupo, M. T. Chemical signaling involved in plant-microbe interactions. Chem Soc Rev 47, 1652-1704, doi:10.1039/c7cs00343a (2018).

5 Dubilier, N., Bergin, C. \& Lott, C. Symbiotic diversity in marine animals: the art of harnessing chemosynthesis. Nat Rev Microbio/ 6, 725-740, doi:Doi 10.1038/Nrmicro1992 (2008).

6 Belin, B. J. et al. Hopanoid lipids: from membranes to plant-bacteria interactions. Nat Rev Microbiol 16, 304-315, doi:10.1038/nrmicro.2017.173 (2018).

$7 \quad$ Kroiss, J. et al. Symbiotic streptomycetes provide antibiotic combination prophylaxis for wasp offspring. Nat Chem Biol 6, 261-263, doi:10.1038/Nchembio.331 (2010).

8 Login, F. H. et al. Antimicrobial Peptides Keep Insect Endosymbionts Under Control. Science 334, 362-365, doi:10.1126/science.1209728 (2011).

9 Finlay, B. B. \& McFadden, G. Anti-immunology: Evasion of the host immune system by bacterial and viral pathogens. Cell 124, 767-782, doi:10.1016/j.cell.2006.01.034 (2006).

10 Nyholm, S. V. \& Graf, J. Knowing your friends: invertebrate innate immunity fosters beneficial bacterial symbioses. Nat Rev Microbiol 10, 815-827, doi:10.1038/nrmicro2894 (2012).

11 Ackermann, M. A functional perspective on phenotypic heterogeneity in microorganisms. Nat Rev Microbiol 13, 497-508, doi:10.1038/nrmicro3491 (2015).

12 Phelan, V. V., Liu, W. T., Pogliano, K. \& Dorrestein, P. C. Microbial metabolic exchange-the chemotype-to-phenotype link. Nat Chem Biol 8, 26-35, doi:10.1038/nchembio.739 (2012).

13 Shank, E. A. Considering the Lives of Microbes in Microbial Communities. Msystems 3, doi:ARTN e00155-17

10.1128/mSystems.00155-17 (2018).

14 Dunham, S. J. B., Ellis, J. F., Li, B. \& Sweedler, J. V. Mass Spectrometry Imaging of Complex Microbial Communities. Accounts Chem Res 50, 96-104, doi:10.1021/acs.accounts.6b00503 (2017).

15 Watrous, J. D. \& Dorrestein, P. C. Imaging mass spectrometry in microbiology. Nat Rev Microbiol 9, 683-694, doi:10.1038/nrmicro2634 (2011).

16 Brunetti, A. E. et al. An integrative omics perspective for the analysis of chemical signals in ecological interactions. Chem Soc Rev 47, 1574-1591, doi:10.1039/c7cs00368d (2018).

17 Kaltenpoth, M., Strupat, K. \& Svatos, A. Linking metabolite production to taxonomic identity in environmental samples by (MA)LDI-FISH. Isme J 10, 527-531, doi:10.1038/ismej.2015.122 (2016).

18 Dorrestein, P. C., Mazmanian, S. K. \& Knight, R. Finding the Missing Links among Metabolites, Microbes, and the Host. Immunity 40, 824-832, doi:10.1016/j.immuni.2014.05.015 (2014).

19 Tropini, C., Earle, K. A., Huang, K. C. \& Sonnenburg, J. L. The Gut Microbiome: Connecting Spatial Organization to Function. Cell Host Microbe 21, 433-442, doi:10.1016/j.chom.2017.03.010 (2017).

20 Passarelli, M. K. et al. The 3D OrbiSIMS-label-free metabolic imaging with subcellular lateral resolution and high mass-resolving power. Nat Methods 14, 1175-+, doi:10.1038/Nmeth.4504 (2017).

21 Amann, R. I. et al. Combination of 16s Ribosomal-Rna-Targeted Oligonucleotide Probes with Flow-Cytometry for Analyzing Mixed Microbial-Populations. Applied and environmental microbiology 56, 1919-1925 (1990).

22 Welch, J. L. M., Hasegawa, Y., McNulty, N. P., Gordon, J. I. \& Borisy, G. G. Spatial organization of a model 15-member human gut microbiota established in gnotobiotic mice. P Natl Acad Sci USA 114, E9105-E9114, doi:10.1073/pnas.1711596114 (2017).

23 Musat, N. et al. A single-cell view on the ecophysiology of anaerobic phototrophic bacteria. $P$ Natl Acad Sci USA 105, 17861-17866, doi:10.1073/pnas.0809329105 (2008). 
24 Dekas, A. E., Poretsky, R. S. \& Orphan, V. J. Deep-Sea Archaea Fix and Share Nitrogen in Methane-Consuming Microbial Consortia. Science 326, 422-426, doi:10.1126/science.1178223 (2009).

25 Soltwisch, J. et al. Mass spectrometry imaging with laser-induced postionization. Science 348, 211-215, doi:10.1126/science.aaa1051 (2015).

26 Zavalin, A., Yang, J., Hayden, K., Vestal, M. \& Caprioli, R. M. Tissue protein imaging at 1 mu m laser spot diameter for high spatial resolution and high imaging speed using transmission geometry MALDI TOF MS. Anal Bioanal Chem 407, 2337-2342, doi:DOI 10.1007/s00216-0158532-6 (2015).

27 Kompauer, M., Heiles, S. \& Spengler, B. Atmospheric pressure MALDI mass spectrometry imaging of tissues and cells at 1.4-mu m lateral resolution. Nat Methods 14, 90-96, doi:10.1038/Nmeth.4071 (2017).

28 Kompauer, M., Heiles, S. \& Spengler, B. Autofocusing MALDI mass spectrometry imaging of tissue sections and 3D chemical topography of nonflat surfaces. Nat Methods 14, 1156-+, doi:10.1038/nmeth.4433 (2017).

29 Spengler, B., Hubert, M. \& Kaufmann, R. in Proceedings of the 42nd Annual Conference on Mass Spectrometry and Allied Topics. 1041 (Chicago).

30 Caprioli, R. M., Farmer, T. B. \& Gile, J. Molecular imaging of biological samples: Localization of peptides and proteins using MALDI-TOF MS. Anal Chem 69, 4751-4760, doi:Doi 10.1021/Ac970888i (1997).

31 Spengler, B. \& Hubert, M. Scanning microprobe matrix-assisted laser desorption ionization (SMALDI) mass spectrometry: instrumentation for sub-micrometer resolved LDI and MALDI surface analysis. J Am Soc Mass Spectrom 13, 735-748, doi:10.1016/S1044-0305(02)00376-8 (2002).

32 Gould, A. L. et al. Microbiome interactions shape host fitness. Proc Natl Acad Sci U SA, doi:10.1073/pnas.1809349115 (2018).

33 Duperron, S. et al. A dual symbiosis shared by two mussel species, Bathymodiolus azoricus and Bathymodiolus puteoserpentis (Bivalvia : Mytilidae), from hydrothermal vents along the northern Mid-Atlantic Ridge. Environ Microbiol 8, 1441-1447, doi:10.1111/j.14622920.2006.01038.x (2006).

34 Szafranski, K. M., Piquet, B., Shillito, B., Lallier, F. H. \& Duperron, S. Relative abundances of methane- and sulfur-oxidizing symbionts in gills of the deep-sea hydrothermal vent mussel Bathymodiolus azoricus under pressure. Deep-Sea Res Pt / 101, 7-13, doi:10.1016/j.dsr.2015.03.003 (2015).

35 Assie, A. et al. A specific and widespread association between deep-sea Bathymodiolus mussels and a novel family of Epsilonproteobacteria. Env Microbiol Rep 8, 805-813, doi:10.1111/1758-2229.12442 (2016).

36 Moreira, D. \& López-García, P. in Encyclopedia of Astrobiology (eds Ricardo Amils et al.) 1-1 (Springer Berlin Heidelberg, 2014).

37 Bemis, K. D. et al. Probabilistic Segmentation of Mass Spectrometry (MS) Images Helps Select Important lons and Characterize Confidence in the Resulting Segments. Molecular \& Cellular Proteomics 15, 1761-1772, doi:10.1074/mcp.0115.053918 (2016).

38 Alexandrov, T. \& Bartels, A. Testing for presence of known and unknown molecules in imaging mass spectrometry. Bioinformatics 29, 2335-2342, doi:10.1093/bioinformatics/btt388 (2013).

39 Burgess, K. E. V., Borutzki, Y., Rankin, N., Daly, R. \& Jourdan, F. MetaNetter 2: A Cytoscape plugin for ab initio network analysis and metabolite feature classification. J Chromatogr $B$ 1071, 68-74, doi:10.1016/j.jchromb.2017.08.015 (2017).

40 Kharbush, J. J., Ugalde, J. A., Hogle, S. L., Allen, E. E. \& Aluwihare, L. I. Composite Bacterial Hopanoids and Their Microbial Producers across Oxygen Gradients in the Water Column of 
the California Current (vol 79, pg 7491, 2013). Applied and environmental microbiology 80 , 3283-3283, doi:10.1128/Aem.00847-14 (2014).

41 Alexandrov, T. et al. METASPACE: A community-populated knowledge base of spatial metabolomes in health and disease. bioRxiv, 539478, doi:10.1101/539478 (2019).

42 Geiger, O., Lopez-Lara, I. M. \& Sohlenkamp, C. Phosphatidylcholine biosynthesis and function in bacteria. Bba-Mol Cell Biol L 1831, 503-513, doi:10.1016/j.bbalip.2012.08.009 (2013).

43 Alvarez, H. M. \& Steinbuchel, A. Triacylglycerols in prokaryotic microorganisms. Appl Microbiol Biot 60, 367-376, doi:10.1007/s00253-002-1135-0 (2002).

44 Yoon, K., Han, D. X., Li, Y. T., Sommerfeld, M. \& Hu, Q. Phospholipid:Diacylglycerol Acyltransferase Is a Multifunctional Enzyme Involved in Membrane Lipid Turnover and Degradation While Synthesizing Triacylglycerol in the Unicellular Green Microalga Chlamydomonas reinhardtii. Plant Cell 24, 3708-3724, doi:10.1105/tpc.112.100701 (2012).

45 Barry, J. P. et al. Methane-based symbiosis in a mussel, Bathymodiolus platifrons, from cold seeps in Sagami Bay, Japan. Invertebr Biol 121, 47-54 (2002).

46 Villarreal-Chiu, J. F., Quinn, J. P. \& McGrath, J. W. The genes and enzymes of phosphonate metabolism by bacteria, and their distribution in the marine environment. Front Microbio/ 3 , doi:Artn 19

\subsection{9/Fmicb.2012.00019 (2012).}

47 Martinez, A., Tyson, G. W. \& DeLong, E. F. Widespread known and novel phosphonate utilization pathways in marine bacteria revealed by functional screening and metagenomic analyses. Environ Microbiol 12, 222-238, doi:10.1111/j.1462-2920.2009.02062.x (2010).

48 Assié, A. et al. Horizontal acquisition of a patchwork Calvin cycle by symbiotic and free-living Campylobacterota (formerly Epsilonproteobacteria). bioRxiv (2018).

49 Esquenazi, E. et al. Visualizing the spatial distribution of secondary metabolites produced by marine cyanobacteria and sponges via MALDI-TOF imaging. Mol Biosyst 4, 562-570, doi:10.1039/b720018h (2008).

50 Simmons, T. L. et al. Biosynthetic origin of natural products isolated from marine microorganism-invertebrate assemblages. P Natl Acad Sci USA 105, 4587-4594, doi:DOI 10.1073/pnas.0709851105 (2008).

51 Wang, M. X. et al. Sharing and community curation of mass spectrometry data with Global Natural Products Social Molecular Networking. Nat Biotechnol 34, 828-837, doi:10.1038/nbt.3597 (2016).

52 Thubaut, J., Puillandre, N., Faure, B., Cruaud, C. \& Samadi, S. The contrasted evolutionary fates of deep-sea chemosynthetic mussels (Bivalvia, Bathymodiolinae). Ecol Evol 3, 47484766, doi:10.1002/ece3.749 (2013).

53 Tavormina, P. L. et al. Methyloprofundus sedimenti gen. nov., sp nov., an obligate methanotroph from ocean sediment belonging to the 'deep sea-1' clade of marine methanotrophs. Int J Syst Evol Micr 65, 251-259, doi:10.1099/ijs.0.062927-0 (2015).

54 Barrero-Canosa, J., Moraru, C., Zeugner, L., Fuchs, B. M. \& Amann, R. Direct-geneFISH: a simplified protocol for the simultaneous detection and quantification of genes and rRNA in microorganisms. Environ Microbiol 19, 70-82, doi:10.1111/1462-2920.13432 (2017).

55 Yamaguchi, T. et al. In situ DNA-hybridization chain reaction (HCR): a facilitated in situ HCR system for the detection of environmental microorganisms. Environ Microbiol 17, 2532-2541, doi:10.1111/1462-2920.12745 (2015).

56 Stewart, G. R., Robertson, B. D. \& Young, D. B. Tuberculosis: A problem with persistence. Nat Rev Microbiol 1, 97-105, doi:10.1038/nrmicro749 (2003).

57 Folkesson, A. et al. Adaptation of Pseudomonas aeruginosa to the cystic fibrosis airway: an evolutionary perspective. Nat Rev Microbiol 10, 841-851, doi:10.1038/nrmicro2907 (2012). 


\section{Methods}

548 Sampling and on-board cryo-fixation. The Bathymodiolus puteoserpentis specimen used for high

549 resolution AP-MALDI-MSI was collected during the RV Meteor M126 cruise in 2016 at the

550 Logatchev hydrothermal vent field on the Mid-Atlantic Ridge. The specimen was retrieved with the

551 MARUM-Quest remotely operated vehicle (ROV) at the Irina II vent site at $3038 \mathrm{~m}$ depth,

552

553

554 $14^{\circ} 45^{\prime} 11.01^{\prime \prime} \mathrm{N}$ and $44^{\circ} 58^{\prime} 43.98^{\prime \prime} \mathrm{W}$, and placed in an insulated container to prevent temperature changes during recovery. Gills were dissected from the mussel as soon as brought on board after ROV retrieval, submerged in precooled $2 \% \mathrm{w} / \mathrm{v}$ carboxymethyl cellulose gel $\left(\mathrm{CMC}, \mathrm{M}_{\mathrm{w}} \sim 700,000\right.$, SigmaAldrich Chemie $\mathrm{GmbH}$ ) and snap-frozen in liquid $\mathrm{N}_{2}$. Samples were stored at $-80{ }^{\circ} \mathrm{C}$ until use. Additional deep-sea mussels from other cruises and sites were snap-frozen but not embedded for APMALDI-MSI (Supplementary Table 5).

Tissue sectioning. The CMC-embedded gills were cross-sectioned at $10 \mu \mathrm{m}$ thickness with a cryostat (Leica CM3050 S, Leica Biosystems Nussloch $\mathrm{GmbH}$ ) at a chamber temperature of $-35^{\circ} \mathrm{C}$ and object holder at $-22{ }^{\circ} \mathrm{C}$. Individual sections were thaw-mounted onto coated Polysine ${ }^{\circledR}$ slides (Thermo Scientific) and subsequently frozen in the cryostat chamber. Slides with tissue sections were stored in slide containers with silica granules, to prevent air moisture condensation on the tissue upon removal from the freezer.

Matrix and landmark application. Before AP-MALDI matrix application, the sample was warmed to room temperature under a dry atmosphere in a sealed slide container (LockMailer ${ }^{\mathrm{TM}}$ microscope slide jar, Sigma-Aldrich, Steinheim, Germany), filled with silica granules (Carl Roth GmbH) to avoid condensation on the cold glass slide. The sample glass slide was marked with white paint around the tissue for orientation during image acquisition as previously described ${ }^{1}$. Additionally, optical images of the tissue section were acquired with a digital microscope (VHX-5000 Series, Keyence, NeuIsenburg, Germany) prior to matrix application (Supplementary Fig. 1). To apply the matrix, we used 
an ultrafine pneumatic sprayer system with $\mathrm{N}_{2}$ gas (SMALDIPrep, TransMIT GmbH, Giessen, Germany) $)^{2}$, to deliver $100 \mu \mathrm{l}$ of a $30 \mathrm{mg} \mathrm{ml}^{-1}$ solution of 2,5-dihydroxybenzoic acid (DHB; $98 \%$ purity, Sigma-Aldrich, Steinheim, Germany) dissolved in acetone/water (1:1 v/v) containing 0.1\% trifluoroacetic acid (TFA). To locate the field of view and facilitate laser focusing, a red marker was applied adjacent to the matrix-covered tissue section.

High (spatial) resolution AP-MALDI-MSI. AP-MALDI-MSI measurements were carried out at an experimental ion source setup ${ }^{2,3}$, coupled to a Fourier transform orbital trapping mass spectrometer (Q Exactive $^{\mathrm{TM}} \mathrm{HF}$, Thermo Fisher Scientific GmbH, Bremen, Germany). The sample was rastered with $233 \times 233$ laser spots with a step size of $3 \mu \mathrm{m}$ without oversampling, resulting in an imaged area of $699 \times 699 \mu \mathrm{m}$ (Supplementary Figs 2 and 3). AP-MALDI-MSI measurements were performed in positive mode for a mass detection range of 400-1200 Da and a mass resolving power of 240,000 (at $200 \mathrm{~m} / \mathrm{z}$ ) (Supplementary Fig. 2). After AP-MALDI-MSI, the measured sample surface was recorded using a stereomicroscope (SMZ25, Nikon, Düssedorf, Germany) (Supplementary Fig. 1).

Magnetic resonance mass spectrometry: Ultra-high mass resolution measurements were carried out with a magnetic resonance mass spectrometer $\left(\operatorname{scimaX}^{\mathrm{TM}}\right.$, Bruker Daltonik $\mathrm{GmbH}$, Bremen, Germany). Samples were extracted with chloroform and the compounds further separated in a methanol washing step in which they stayed in the methanol fraction. These methanol extracts of the samples were measured by electrospray ionization in positive ion mode by direct syringe infusion in a mass range of 107-3000 Da using quadrupolar detection and a mass resolving power of 650,000 (at $400 \mathrm{~m} / \mathrm{z}$ ). The MS and MS/MS spectra were externally calibrated with NaTFA cluster. The MS spectra were additionally internally calibrated with Hexakis $(1 \mathrm{H}, 1 \mathrm{H}, 3 \mathrm{H}$-tetrafluoropropoxy) phosphazene (Apollo Scientific Ltd., compound PC0874). The molecular formula of detected compounds and fragments were determined with DataAnalysis 5.1 (Bruker Daltonik GmbH, Bremen, Germany) (Supplementary table 9). 
600 Fluorescence in situ hybridization. The glass slide with the matrix-covered tissue section was

601

602

603

604

605

606

607

608

609

610

611

612

613

614

615

616

617

618

submerged in a 2\% PFA/PBS (137 mM NaCl, $\left.2.7 \mathrm{mM} \mathrm{KCl,} 10 \mathrm{mM} \mathrm{Na} 2 \mathrm{HPO}_{4}, 2 \mathrm{mM} \mathrm{KH}_{2} \mathrm{PO}_{4}\right)$

solution for one hour at room temperature, to wash off the matrix and stabilize the tissue. The fixation was followed by two 20 min washing steps in PBS, by carefully dipping the slide in $96 \%$ EtOH and air drying. The dried section was encircled with a liquid blocker (PAP-Pen, Science Services) on the glass slide to prevent leakage of the hybridization mixture during incubation ${ }^{4}$.

The hybridization mixture, modified after ${ }^{5}$, contained $5 \mathrm{ng} \mathrm{l}^{-1}$ of probe in hybridization buffer (35\% formamide (v/v), $\mathrm{NaCl} 900 \mathrm{mM}, 20 \mathrm{mM}$ Tris- $\mathrm{HCl}(\mathrm{pH} 7.5), 10 \%$ dextran sulfate (w/v), $0.02 \%(\mathrm{w} / \mathrm{v})$ sodium dodecyl sulfate (SDS), $1 \%$ (w/v) Blocking Reagent (Roche, Basel, Switzerland)). We used specific 16S rRNA probes to target symbiotic SOX (BMARt_193: 5'-CGAAGGTCCTCCACTTTA3') and MOX (BNMARm_845: 5' -GCTCCGCCACTAAGCCTA-3') bacteria ${ }^{6}$. BNMARm_845

contained one Cyanine 3 (Cy3) fluorophore at the 5'-end of the oligonucleotide, and BMARt_193 contained one MFP-ATTO488 fluorophore at each end for increased sensitivity (biomers.net GmbH, Ulm, Germany) ${ }^{7,8}$. A negative control with nonspecific binding (Non338: 5'-ACTCCTACGG-

GAGGCAGC-3' $)^{9}$, labeled with Cy3, was hybridized on a subsequent tissue section during the same FISH experiment.

Tissue sections of sample and controls were hybridized with $20 \mu \mathrm{l}$ of hybridization mixture, for $2 \mathrm{~h}$ in a saturated formamide-water atmosphere at $46{ }^{\circ} \mathrm{C}$. Washing of the samples was conducted as described in ${ }^{6}$. Subsequently, the DNA of host and symbiotic bacteria was stained with 4',6-diamidino2-phenylindole (DAPI) for $3 \times 10 \mathrm{~min}$ at room temperature. For microscopy, sections were mounted with VECTASHIELD ${ }^{\circledR}$.

Fluorescence microscopy. Overview tile-scans $(0.32 \mu \mathrm{m} / \mathrm{pixel})$ of the sample and the control were first acquired in the bright field and fluorescent channels with an automated epifluorescence microscope (Zeiss Axio Imager Z2.m, AxioCam MRm, Plan-Neofluar 20x/0.8) operated by a tilescanning macro for Axio Vision (v. SE64 4.9.1, Carl Zeiss Microscopy GmbH, Germany). 

objective lens.

To cover a larger area with the CLSM than measured with AP-MALDI-MSI a region of 25 tiles $(5 \times$ 5) with each tile covering $425.1 \mu \mathrm{m} \times 425.1 \mu \mathrm{m}(0.21 \mu \mathrm{m} /$ pixel $)$ and $4 z$ layers with $5.72 \mu \mathrm{m}$ per layer. The area measured with AP-MALDI-MSI $(699 \mu \mathrm{m} \times 699 \mu \mathrm{m})$ overlapped with 6 tiles $(3 \times 2)$ of the CLSM scan, which were used for further analysis. The FISH signals were recorded from one focal plane, which was used for further processing and the correlative analysis (Supplementary Fig. 3). The tiles were stitched with ZEN black (v. 14.0.1.201, Carl Zeiss Microscopy GmbH, Germany) and readjusted with the MosaicJ plugin in imagej (v. 1.50d) (Supplementary Fig. 3).

642

643 Correlative image processing. Alignment of AP-MALDI-MSI and FISH imaging data was performed in Matlab R2016a (Supplementary text 1). Before alignment, the MSI imaging dataset was inflated to the microscopy pixel size to prevent loss of structural information in the microscopy image. representative of the tissue structure (Supplementary Fig. 8). The transformation was based on 10 corresponding landmarks on the tissue on the RGB microscopy image and the MSI consensus image. intensities for the three 8-bit single-channel images (RGB) were exported as an Excel table for further 
processing in R. For further representation, we used CMY instead of RGB as color code. To determine the threshold between noise and fluorescent probe signal, we evaluated the threshold between signals and noise for each channel using the image segmenting app in Matlab. This grey value threshold was then used in the R pipeline. To represent an overall tissue signal, the bright field microscopy image from the overview tile-scan was aligned to the CLSM image (Supplementary Figs 1 and 8), segmented and transformed into a binary matrix to define "on tissue" and "off tissue" regions to perform background removal of MSI pixels (Supplementary Text 2 and Supplementary Fig. 8).

AP-MALDI-MSI data preprocessing. After imaging, the Thermo *.raw files were centroided and converted to *.mzML with MSConvert GUI (ProteoWizard, v. 3.0.9810, ${ }^{11}$ ) and then converted to *.imZML using the imZML Converter $1.3^{12}$. The *.imZML MALDI-MSI data was imported into R (v. 3.4.0, Supplementary Text 2) and processed using the Cardinal package (v. 1.8.0, ${ }^{13}$ ). Briefly, data was imported using the readMSIData function with a mass accuracy of 1 . The raw data was normalized to the total ion chromatogram and peak picking carried out using the adaptive method with a signal to noise ratio of 10 .

Correlative AP-MALDI-MSI and FISH data analysis. Pixel-aligned FISH signal matrices of all three fluorescent channels (DAPI, SOX, MOX) were imported into R. Of the 255 greyscale values, background noise pixels were defined for intensities from 0 to 20 (see above for the choice of threshold). Pixels of each image matrix for the MOX, SOX and DAPI channels with intensities from 21 to 255 were counted as signal.

The host-only tissue area was determined by subtracting the MOX and SOX symbiont pixels from the DAPI pixels. Furthermore, we used the "on/off-tissue" bright-field microscopy signal matrix to remove background pixels in AP-MALDI-MSI data (Supplementary Fig. 8). Thereby, we reduced data size and minimized the influence of background signals on the downstream analysis. To further reduce data dimensionality, $\mathrm{m} / \mathrm{z}$ values were only retained if a peak was present in at least $10 \%$ of the pixel area of the spatially smallest fluorescent channel. 
Spatial cluster analysis was performed on the reduced data using the spatial shrunken centroids (ssc) method, which performed unsupervised segmentation of the MSI dataset (Supplementary Fig. 6) ${ }^{14}$. This technique allowed us to select for the best-fit model of the appropriate number of metabolite clusters $(k)$ and informative metabolite features (Supplementary Fig. 5). We generated a Bray-Curtis dissimilarity matrix with the vegdist function in the vegan package ${ }^{15}$ from the mean spectra of the ssc cluster groups. We then used this dissimilarity matrix to visualize the similarity between clusters in a hierarchical tree (Fig. 2c, Supplementary Fig. 5, 6). Using both matrices of the aligned FISH signals and the cluster segmentation maps, we calculated the area overlapto define the percentage of fluorescent bacteria- and host signals per cluster (Supplementary Text 2 and 4).

Metabolite annotations of AP-MALDI-MSI data. Identification of metabolites from AP-MALDIMSI data was carried out with bioinformatic approaches using exact mass and isotope ratio matches of known metabolites from the databases $\mathrm{HMBD}^{16}, \mathrm{ChEBI}^{17}$ and The LIPID MAPS Lipidomics Gateway (http://www.lipidmaps.org/). In addition, selected metabolites were analyzed via on-tissue MS2 and fragmentation pattern comparison and uploaded to the automated annotation platform Metaspace ${ }^{18,19}$. Putative annotations and their scoring were exported as a *.csv list for further analysis.

Molecular networking. Molecular networks were visualized in Cytoscape (v. 3.5.1) ${ }^{20}$, the APMALDI-MSI MS1 data with the MetaNetter $2^{21}$ and the LC-MS/MS data with GNPS ${ }^{22}$. Community matching, dereplication and metabolite annotation of the network based on LC-MS/MS were conducted with the default settings of the respective GNPS pipelines (Supplementary Text 3). The MSI peak list was imported to create nodes, and a list with major chemical transformations without isotopes and matrix adducts was used to calculate the mass differences as edges between the nodes with Metanetter 2 (Supplementary Table S2). The networks were then created with the Allegro Layout application (v. 2.2.2) to avoid overlapping of nodes and to adjust the node to edge length ratio for visualization. Coloring and reshaping of nodes was performed in Cytoscape using the cluster and annotation data. 
Solvents for LC-MS/MS. All organic solvents were LC-MS grade, using acetonitrile (ACN;

709

710

711

712

713

714

715

716

717

718

719

720

721

722

723

724

725

726

Honeywell, Honeywell Specialty Chemicals Seelze GmbH), isopropanol (IPA; BioSolve,

Valkenswaard, The Netherlands), and formic acid (FA; Sigma-Aldrich Biochemie GmbH Hamburg).

Water was deionized by using the Astacus MembraPure system (MembraPure GmbH, Henningsdorf, Berlin, Germany).

Lipid extraction for LC-MS/MS. Lipids were extracted from small pieces of frozen gills (50-100 mg) using a mixture of $\mathrm{ACN}$, methanol $(\mathrm{MeOH})$ and water $\left(\mathrm{H}_{2} \mathrm{O}\right)(2: 2: 1 \mathrm{v} / \mathrm{v} / \mathrm{v})$, by bead beating using a stainless steel bead (FastPrep®-24, MP) for $2 \times 40 \mathrm{~s}$ at $4 \mathrm{~m} / \mathrm{s}$. The tissues were then centrifuged (2 min, $15,600 \mathrm{~g}, 4^{\circ} \mathrm{C}$ ) and the supernatant transferred into HPLC vials for analysis.

High resolution LC-MS/MS. The analysis was performed using a QExactive Plus Orbitrap (Thermo Fisher Scientific) equipped with an HESI probe and a Vanquish Horizon UHPLC System (Thermo Fisher Scientific). The lipids were separated on an Accucore C30 column $(150 \times 2.1 \mathrm{~mm}, 2.6 \mu \mathrm{m}$, Thermo Fisher Scientific), at $40{ }^{\circ} \mathrm{C}$, using a solvent gradient. Buffer A (60/40 ACN/ $\mathrm{H}_{2} \mathrm{O}, 10 \mathrm{mM}$ ammonium formate, 0.1\% FA) and buffer B (90/10 IPA/ACN, $10 \mathrm{mM}$ ammonium formate, $0.1 \%$ FA) $)^{23}$ were used at a flow rate of $350 \mu 1 \mathrm{~min}^{-1}$. The lipids were eluted from the column with a gradient starting at $0 \%$ buffer B (Supplementary Table 6).The injection volume was $10 \mu$ l. In the same run, MS measurements were acquired in positive and negative mode for a mass detection range of $150-1500$

Da (Supplementary Table 7). The resolution of the mass analyzer was set to 70,000 for MS1 scans and 35,000 for MS2 scans at $200 \mathrm{~m} / \mathrm{z}$. MS/MS scans of the eight most abundant precursor ions were acquired in positive and negative modes. Dynamic exclusion was enabled for 30 seconds and collision energy was set to $30 \mathrm{~V}$.

Data availability. Download links for the raw files of fluorescence microscopy data, MALDI-MSI and on-tissue MALDI-MS/MS data and LC-MS/MS data are provided in the Supplementary Text 3. 
Data, analyzed with the online annotation and networking platforms Metaspace and GNPS can also be

accessed through the provided links in the Supplementary Text 3.

\section{References}

$1 \quad$ Kaltenpoth, M., Strupat, K. \& Svatos, A. Linking metabolite production to taxonomic identity

2 Kompauer, M., Heiles, S. \& Spengler, B. Atmospheric pressure MALDI mass spectrometry in environmental samples by (MA)LDI-FISH. Isme J 10, 527-531, doi:10.1038/ismej.2015.122 (2016).

imaging of tissues and cells at 1.4-mu m lateral resolution. Nat Methods 14, 90-96, doi:10.1038/Nmeth.4071 (2017).

3 Kompauer, M., Heiles, S. \& Spengler, B. Autofocusing MALDI mass spectrometry imaging of tissue sections and 3D chemical topography of nonflat surfaces. Nat Methods 14, 1156-+, doi:10.1038/nmeth.4433 (2017).

4 Duperron, S. et al. Dual symbiosis in a Bathymodiolus sp mussel from a methane seep on the gabon continental margin (southeast Atlantic): 16S rRNA phylogeny and distribution of the symbionts in gills. Applied and environmental microbiology 71, 1694-1700, doi:Doi 10.1128/Aem.71.4.1694-1700.2005 (2005).

5 Pernthaler, A., Pernthaler, J. \& Amann, R. Fluorescence in situ hybridization and catalyzed reporter deposition for the identification of marine bacteria. Applied and environmental microbiology 68, 3094-3101, doi:Doi 10.1128/Aem.68.6.3094-3101.2002 (2002).

6 Duperron, S. et al. A dual symbiosis shared by two mussel species, Bathymodiolus azoricus and Bathymodiolus puteoserpentis (Bivalvia : Mytilidae), from hydrothermal vents along the northern Mid-Atlantic Ridge. Environ Microbiol 8, 1441-1447, doi:10.1111/j.14622920.2006.01038.x (2006).

7 Amann, R. I. et al. Combination of 16s Ribosomal-Rna-Targeted Oligonucleotide Probes with Flow-Cytometry for Analyzing Mixed Microbial-Populations. Applied and environmental microbiology 56, 1919-1925 (1990).

8 Stoecker, K., Dorninger, C., Daims, H. \& Wagner, M. Double Labeling of Oligonucleotide Probes for Fluorescence In Situ Hybridization (DOPE-FISH) Improves Signal Intensity and Increases rRNA Accessibility. Applied and environmental microbiology 76, 922-926, doi:10.1128/Aem.02456-09 (2010).

9 Wallner, G., Amann, R. \& Beisker, W. Optimizing Fluorescent Insitu Hybridization with Ribosomal-Rna-Targeted Oligonucleotide Probes for Flow Cytometric Identification of Microorganisms. Cytometry 14, 136-143, doi:DOI 10.1002/cyto.990140205 (1993).

10 Verbeeck, N. et al. Connecting imaging mass spectrometry and magnetic resonance imagingbased anatomical atlases for automated anatomical interpretation and differential analysis. Bba-Proteins Proteom 1865, 967-977, doi:10.1016/j.bbapap.2017.02.016 (2017).

11 Chambers, M. C. et al. A cross-platform toolkit for mass spectrometry and proteomics. Nat Biotechnol 30, 918-920, doi:10.1038/nbt.2377 (2012).

12 Race, A. M., Styles, I. B. \& Bunch, J. Inclusive sharing of mass spectrometry imaging data requires a converter for all. J Proteomics 75, 5111-5112, doi:10.1016/j.jprot.2012.05.035 (2012).

13 Bemis, K. D. et al. Cardinal: an R package for statistical analysis of mass spectrometry-based imaging experiments. Bioinformatics 31, 2418-2420, doi:10.1093/bioinformatics/btv146 (2015). 
14 Bemis, K. D. et al. Probabilistic Segmentation of Mass Spectrometry (MS) Images Helps Select Important lons and Characterize Confidence in the Resulting Segments. Mol Cell Proteomics 15, 1761-1772, doi:10.1074/mcp.0115.053918 (2016).

15 Dixon, P. VEGAN, a package of R functions for community ecology. J Veg Sci 14, 927-930, doi:DOI 10.1111/j.1654-1103.2003.tb02228.x (2003).

16 Wishart, D. S. et al. HMDB 4.0: the human metabolome database for 2018. Nucleic Acids Res 46, D608-D617, doi:10.1093/nar/gkx1089 (2018).

17 Hastings, J. et al. ChEBI in 2016: Improved services and an expanding collection of metabolites. Nucleic Acids Res 44, D1214-D1219, doi:10.1093/nar/gkv1031 (2016).

18 Palmer, A. et al. FDR-controlled metabolite annotation for high-resolution imaging mass spectrometry. Nat Methods 14, 57-60, doi:10.1038/Nmeth.4072 (2017).

19 Alexandrov, T. et al. METASPACE: A community-populated knowledge base of spatial metabolomes in health and disease. bioRxiv, 539478, doi:10.1101/539478 (2019).

20 Shannon, P. et al. Cytoscape: A software environment for integrated models of biomolecular interaction networks. Genome Res 13, 2498-2504, doi:10.1101/gr.1239303 (2003).

21 Burgess, K. E. V., Borutzki, Y., Rankin, N., Daly, R. \& Jourdan, F. MetaNetter 2: A Cytoscape plugin for ab initio network analysis and metabolite feature classification. J Chromatogr $B$ 1071, 68-74, doi:10.1016/j.jchromb.2017.08.015 (2017).

22 Wang, M. X. et al. Sharing and community curation of mass spectrometry data with Global Natural Products Social Molecular Networking. Nat Biotechnol 34, 828-837, doi:10.1038/nbt.3597 (2016).

23 Breitkopf, S. B. et al. A relative quantitative positive/negative ion switching method for untargeted lipidomics via high resolution LC-MS/MS from any biological source.

Metabolomics 13, doi:ARTN 30

10.1007/s11306-016-1157-8 (2017). 